\title{
BMJ Open What resources are used in emergency departments in rural sub-Saharan Africa? A retrospective analysis of patient care in a district-level hospital in Uganda
}

Cindy Carol Bitter, ${ }^{1,2}$ Brian Rice, ${ }^{2,3}$ Usha Periyanayagam, ${ }^{2,4}$ Bradley Dreifuss, ${ }^{2,5,6}$ Heather Hammerstedt, ${ }^{2,7}$ Sara W Nelson, ${ }^{2,8}$ Mark Bisanzo, ${ }^{2,9}$ Samuel Maling, ${ }^{10}$ Stacey Chamberlain ${ }^{2,11}$

To cite: Bitter CC, Rice B, Periyanayagam $\mathrm{U}$, et al. What resources are used in emergency departments in rural sub-Saharan Africa? $A$ retrospective analysis of patient care in a district-level hospital in Uganda. BMJ Open 2018;8:e019024. doi:10.1136/ bmjopen-2017-019024

- Prepublication history for this paper is available online. To view these files, please visit the journal online $\mathrm{http} / / / \mathrm{dx}$.doi. org/10.1136/bmjopen-2017019024).

Received 27 August 2017 Revised 9 November 2017 Accepted 14 November 2017

Check for updates

For numbered affiliations see end of article.

Correspondence to

Dr Mark Bisanzo;

mbisanzo@gmail.com

\section{ABSTRACT}

Objectives To determine the most commonly used resources (provider procedural skills, medications, laboratory studies and imaging) needed to care for patients.

Setting A single emergency department (ED) of a districtlevel hospital in rural Uganda.

Participants 26710 patient visits.

Results Procedures were performed for $65.6 \%$ of patients, predominantly intravenous cannulation, wound care, bladder catheterisation and orthopaedic procedures. Medications were administered to $87.6 \%$ of patients, most often pain medications, antibiotics, intravenous fluids, antimalarials, nutritional supplements and vaccinations. Laboratory testing was used for $85 \%$ of patients, predominantly malaria smears, rapid glucose testing, HIV assays, blood counts, urinalyses and blood type. Radiology testing was performed for $17.3 \%$ of patients, including X-rays, point-of-care ultrasound and formal ultrasound. Conclusion This study describes the skills and resources needed to care for a large prospective cohort of patients seen in a district hospital ED in rural sub-Saharan Africa. It demonstrates that the vast majority of patients were treated with a small formulary of critical medications and limited access to laboratories and imaging, but providers require a broad set of decision-making and procedural skills.

\section{INTRODUCTION}

Improving the delivery of acute care services has been proposed as a means to strengthen overall health infrastructure by supplementing primary care and reducing fragmentation caused by targeting markers of specific disease processes for improvement. ${ }^{1}$ Global research in the development of systems for acute and emergency care is hampered by the variety of settings in which emergency care takes place in different parts of the world, poor understanding of the reasons patients
Strengths and limitations of this study

- The study follows a large, multiyear cohort which accounts for seasonal variation in disease prevalence.

- Data reflect local resource limitations; additional tests and imaging may have been helpful to optimise patient outcomes.

- No assessment was made of the appropriateness of procedures and testing, or whether additional studies might have been indicated.

seek emergency care, poor standardisation of reporting measures and lack of consensus on the essential services that should be provided. ${ }^{2}$ The African Federation for Emergency Medicine (AFEM) has proposed a framework for emergency care development in the region. ${ }^{3}$ However, there are few longitudinal evaluations of facility-level data that assess the epidemiology of patient presentations, the consumable and non-consumable resources used for care and the clinical and procedural skills required to deliver emergency care in sub-Saharan Africa (SSA) on which to base these efforts. ${ }^{4-7}$ The limited data that exist are largely from urban areas or describe presentations over a short time frame, introducing possibility of bias, given the seasonal nature of some diseases thought to be prevalent in this area. It is estimated that $62 \%$ of the population of SSA lives in rural areas, ${ }^{8}$ indicating that data from rural areas are essential to guide emergency care development.

The uncategorised burden of disease likely contributes to the lack of funding and slow progress of development of emergency care in these countries. ${ }^{9}$ While several emergency medicine training programmes have been 
developed over the past decade ${ }^{10-15}$ and the AFEM has developed a curriculum for emergency care training, ${ }^{16}$ the educational content of these efforts has largely been drawn from international guidelines and expert consensus of foreign emergency physicians and local physicians providing emergency care in these settings.

Lack of information regarding the most frequently used procedural skills and the resources required to care for acutely ill and injured patients hinders the development of emergency care delivery and training in low-resource settings. This study describes the resources and clinical skills used to care for a large, longitudinal cohort of patients presenting to a not-for-profit district hospital in rural Uganda. It addresses some of the research gaps in service delivery, resource utilisation and training needs necessary to ensure effective emergency care as identified in a recent consensus statement. ${ }^{17}$ This knowledge could inform required training and protocols, emergency care formulary development and checklists of useful laboratory resources and diagnostic imaging modalities to refine the proposed framework for emergency care development in SSA. ${ }^{18}$

\section{METHODS}

\section{Study design}

This is a retrospective analysis of prospectively collected data from a quality assurance (QA) database of patients seen at the Karoli Lwanga Hospital Emergency Department (ED) from November 2009 through February 2015. The database includes demographic information obtained on arrival in the ED, as well as diagnostic studies, medications administered and procedures performed during ED care. Laboratories, imaging and procedures ordered in the ED but delayed until the patient was admitted to the ward were included, so the numbers reflect the resources that providers felt were needed to care for the patients, regardless of whether the resources were immediately available in the ED.

\section{Background and study setting}

Karoli Lwanga Hospital is a private, not-for-profit district hospital located in the town of Nyakibale, in the Rukungiri district of rural Southwest Uganda. The district covers $1445 \mathrm{~km}^{2}$, and the 2012 census listed 321300 persons. Life expectancy is 50 years, compared with a national figure of 58.7 years. The HIV prevalence rate is $8.7 \%$ compared with a national rate of $7.2 \%$. The maternal mortality rate is 474 per 100000 live births, compared with a national rate of 440 per 100000 . The infant mortality rate is 76 per 1000 live births, compared with national rate of 45 per 1000. There are two district hospitals in the area, 63 health centres and 17 private clinics. The nearest referral hospital is in Mbarara, some $110 \mathrm{~km}$ away from Nyakibale. ${ }^{19} 20$

Karoli Lwanga Hospital has a six-bed ED with an average census of 500 patients per month. The ED is located centrally in the hospital, adjacent to most wards.
The outpatient department (OPD) operates from 09:00 to 17:00 Monday through Friday and from 08:00 to 12:00 on Saturday. Ambulatory patients are informally triaged, usually by a nurse or clinical officer, and may be referred to the ED based on chief complaint or concerning signs or symptoms noted on patient evaluation. All patients thought to require hospital admission are sent from the OPD to the ED. In addition, patients who are non-ambulatory, severely ill or acutely injured are seen in the ED directly, as are those arriving during hours when OPD is closed. The ED hours varied during the time of the study, but always began at 08:00 and extended until at least 22:00. For the past several years, the ED sees patients between 08:00 and 02:00. For emergency patients arriving during overnight hours when the ED is closed, hospital security would phone the on-call physician or clinical officer. These patients are not included in this study; however, during a 6-month period when the ED was open 24 hours a day, only two patients on average arrived between 02:00 and 08:00 per day.

The ED is staffed by non-physician clinicians locally known as emergency care practitioners (ECPs); these are nurses who are enrolled in or have completed a 2-year training programme in emergency care. The training programme was developed by a US-based non-profit organisation, Global Emergency Care, that has been working to expand emergency care services in Uganda since 2008, in collaboration with Karoli Lwanga Hospital, Mbarara University of Science and Technology and the Ugandan Ministry of Health. During the period of the study, ECPs transitioned from full-time supervision by Emergency Medicine board-certified/board eligible physicians to more independent patient care with supervision by senior-level ECPs and intermittent supervision by EM physicians. This training programme and care delivery model have been described in more depth elsewhere. ${ }^{14}{ }^{21}$ Hospital-based Ugandan physicians were on-call for consultation for acute surgical emergencies and severely ill patients requiring admission.

\section{Data collection and analysis}

The study population includes all ED patient visits from 14 November 2009 to 28 February 2015. The start date reflects the initial day that data were collected on ED patients, which was approximately 4 months after the initial training of the ECPs began. Orders were placed by ECPs on a structured handwritten chart; this information was prospectively entered into the QA database by a trained research assistant at the time of ED disposition. Additionally, during most of the time period, the paper charts were scanned and securely saved as part of the QA processes. The QA database tracked 31 fields including demographics, vital signs, chief complaint, laboratory studies, imaging, medications administered, procedures performed, final diagnosis, disposition and condition on discharge. As this is the first programme to use task shifting for provision of emergency care in SSA, the QA database was created to monitor patient 


\begin{tabular}{|c|c|c|}
\hline & Number & Per cent \\
\hline \multicolumn{3}{|l|}{ Sex } \\
\hline Male & 14720 & 55.1 \\
\hline Female & 11934 & 44.7 \\
\hline Unspecified & 56 & 0.2 \\
\hline \multicolumn{3}{|l|}{ Age } \\
\hline Children under 5 years & 5478 & 20.5 \\
\hline Paediatric 5-17 years & 4337 & 16.2 \\
\hline Adult 18-65years & 13510 & 50.6 \\
\hline Elderly $>65$ years & 3305 & 12.4 \\
\hline
\end{tabular}

presentations and outcomes, to better characterise acute care needs and to improve programme operations and educational content.

Data collection initially used Microsoft Excel (Microsoft, Redmond, Washington, USA) and was transitioned to Microsoft Access in March 2012. The database was deidentified prior to analysis. Data were merged and analysed in Stata Statistical Software V.13 (Stata Statistical Software: Release 13). The data were formatted and cleaned, and the variables of interest were abstracted from the general database prior to analysis by a single researcher. Descriptive tables of the most common skills and resources were created.

\section{RESULTS}

The cohort includes a total of 26710 patient visits. Males accounted for 14720 (55.1\%) visits. Paediatric patients under 5 years of age were $20.5 \%$ of total visits, and patients aged $5-17$ were $16.2 \%$ of visits. Adults $18-65$ years accounted for $50.6 \%$ of visits, and elders over 65 accounted for $12.4 \%$ of visits (table 1 ).

One or more procedures were performed during 17509 $(65.6 \%)$ patient visits, with a total of 22729 procedures performed. Multiple procedures were required for 3601 (14.8\%) patient visits with a mean of 1.3 and a maximum of seven procedures among those who required a procedure. The most common procedure performed was intravenous cannulation. Wound care procedures were next, followed by bladder catheterisation, splinting and immobilisation, procedural sedation, lumbar punctures and incision and drainage of abscesses (I\&D) (table 2).

A total of 73317 doses of medications were administered. Medications were used for 23401 (87.6\%) patient visits, with $20705(77.5 \%)$ receiving more than one medication. The most common class of medications used was analgesics, including acetaminophen, non-steroidal anti-inflammatories and opioid analgesics. The next most common class prescribed was antibiotics, followed by antimalarials, intravenous fluids and nutritional supplements. Approximately 1800 medications could not be classified due to misspellings or non-standard abbreviations. Medication categories are listed in table 3, and the
Table 2 Procedures

\begin{tabular}{|c|c|c|c|}
\hline Procedure & Number & $\begin{array}{l}\text { Per cent of } \\
\text { procedures }\end{array}$ & $\begin{array}{l}\text { Per cent of } \\
\text { cohort }\end{array}$ \\
\hline Intravenous & 9161 & 40.3 & 34.2 \\
\hline Wound care & 7246 & 31.9 & 27.1 \\
\hline Catheter & 1523 & 6.7 & 5.7 \\
\hline $\begin{array}{l}\text { Splint and } \\
\text { immobilisation }\end{array}$ & 743 & 3.3 & 2.8 \\
\hline $\begin{array}{l}\text { Procedural } \\
\text { sedation }\end{array}$ & 663 & 2.9 & 2.5 \\
\hline Lumbar puncture & 513 & 2.3 & 2.0 \\
\hline $\begin{array}{l}\text { Incision and } \\
\text { Drainage }\end{array}$ & 511 & 2.2 & 1.9 \\
\hline
\end{tabular}

most commonly used medications from each category are listed in table 4 . Specific medications given to more than $0.5 \%$ of the patient cohort are listed in table 5 .

Laboratory tests were ordered for $22708(85.0 \%)$ patient visits, with a total of 38378 studies being performed. The most common study was malaria testing, done in 14440 $(54.0 \%)$ patient visits. Bedside glucose was checked in $5666(21.2 \%)$ patient visits. HIV testing was done in 5195 (19.4\%) patient visits with CD4 testing for 465 (1.7\%). Haemoglobin was run for 4108 (15.4\%) patient visits. Urinalysis was performed for $3062(11.5 \%)$ patient visits. Blood type was checked for $1366(5.1 \%)$ patient visits (table 6).

Radiology studies were performed for 4630 (17.3\%) patient visits. X-rays were performed or ordered on 1864 $(7.0 \%)$ patient visits. The most common X-ray was a chest $\mathrm{X}$-ray, done for 1071 patient visits $(4.0 \%$ of the cohort, $45.6 \%$ of all X-rays requested.) Other X-rays included 477 lower and 275 upper extremity films, 89 pelvis films, 88 skull films and 73 abdominal films. X-rays were available only when a radiology technician was present, mostly weekday business hours, but with some call-in capacity on evenings and weekends. Point-of-care ultrasound (POCUS) was performed on 2194 (8.2\%) patient visits

Table 3 Medications by category

\begin{tabular}{lll}
\hline Medication class & Doses & $\begin{array}{l}\text { Per cent of } \\
\text { medications given }\end{array}$ \\
\hline Analgesics & 22981 & 31.4 \\
Antibiotics & 14486 & 19.8 \\
\hline Antimalarials & 5495 & 7.5 \\
\hline Intravenous fluids & 5445 & 7.4 \\
\hline Nutritional & 4785 & 6.5 \\
\hline Respiratory & 3394 & 4.6 \\
\hline Gastrointestinal & 2584 & 3.5 \\
Neurologic & 2495 & 3.4 \\
Cardiac & 2489 & 3.4 \\
Vaccines & 1984 & 2.7 \\
\hline
\end{tabular}


Table 4 Most common medications by category

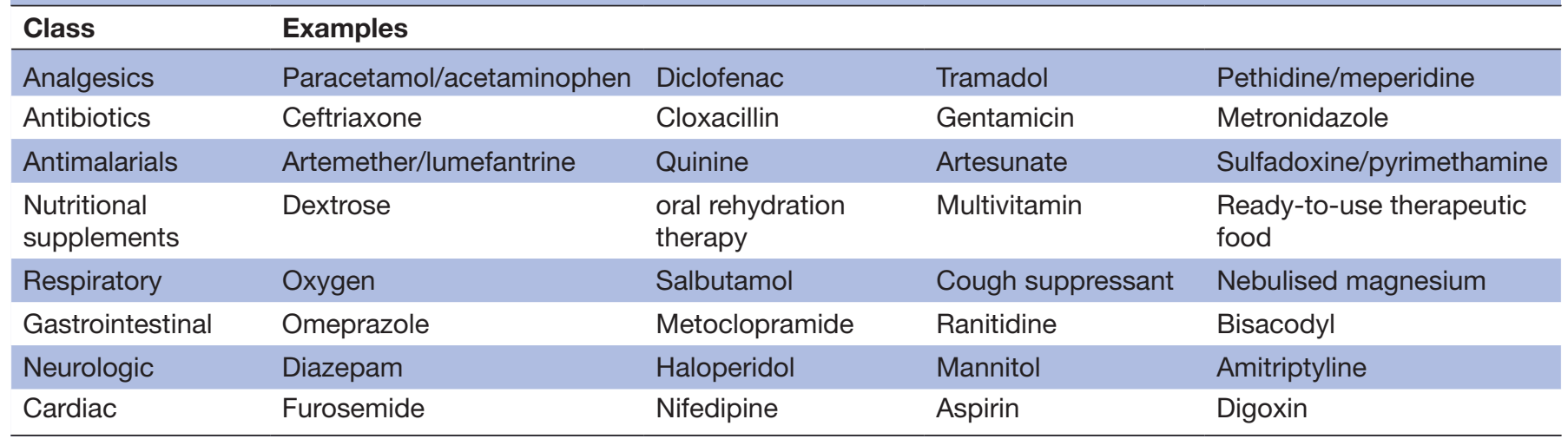

including 417 focused assessment with sonography in trauma (FAST) examinations, 139 abdominal studies, 107 echocardiograms and 73 transabdominal examinations for obstetrical or gynaecological indications. Formal ultrasound imaging by the hospital radiology technician was requested for 998 (3.7\%) patient visits (table 7).

\section{DISCUSSION}

This is a longitudinal cohort of 26710 acutely ill patient visits cared for in a rural emergency department in SSA. The study builds on the work of previous authors in several important ways. First, to our knowledge, this is the largest data set of emergencies presenting to a rural district hospital in a low-resource country. Additionally, we report detailed information on procedures performed and resources used for care in this large cohort over a 5-year period. To deliver effective emergency care, ongoing research is required to further elucidate both training and resource needs in resource-limited settings. By documenting current practice patterns of emergency care in a functional ED in rural Uganda, the data could inform training and resource allocation for providers in similar settings.

\begin{tabular}{lll}
\hline \multicolumn{2}{l}{ Table 5} & Most common medications by \\
\hline Medication & Doses & $\begin{array}{l}\text { Per cent of } \\
\text { medications }\end{array}$ \\
\hline Paracetamol & 14697 & 20.0 \\
\hline Ceftriaxone & 4697 & 6.41 \\
\hline Diclofenac & 4508 & 6.2 \\
\hline Normal saline & 3672 & 5.0 \\
\hline Coartem & 3542 & 4.8 \\
\hline Tetanus vaccine & 1862 & 2.5 \\
\hline Tramadol & 1842 & 2.5 \\
Quinine & 1775 & 2.4 \\
Cloxacillin & 1686 & 2.3 \\
Gentamicin & 1602 & 2.2 \\
Ringer's lactate & 1446 & 2.0 \\
\hline
\end{tabular}

In considering medical knowledge that should be included in emergency care training, the spectrum of diseases presenting acutely must be understood. Data from the Global Burden of Disease Study indicate an increasing contribution of non-communicable disease towards worldwide mortality, ${ }^{22}$ but it is unknown how much these diseases contribute to the burden of emergencies in SSA. The data show that ECPs cared for patients across all age ranges who presented with diverse complaints. Commonly used medications and diagnostic tests indicate that patients presented for emergency care with a broad array of communicable and non-communicable diseases. Our data suggest that even in rural areas with high rates of communicable diseases, non-communicable diseases contribute significantly to the need for acute care. Additionally, given the fact that analgesics were given to $31.4 \%$ of patients and approximately $2.5 \%$ of patients underwent procedural sedation, it can be inferred that the ECPs managed a large burden of acutely painful conditions. High rates of splinting and immobilisation, as well as the number of FAST examinations performed, indicate that trauma care is a prominent component of emergency care in this setting. This is

Table 6 Laboratory testing

\begin{tabular}{lll}
\hline Medication & Doses & $\begin{array}{l}\text { Per cent of } \\
\text { medications }\end{array}$ \\
\hline Paracetamol & 14697 & 20.0 \\
Ceftriaxone & 4697 & 6.41 \\
Diclofenac & 4508 & 6.2 \\
\hline Normal saline & 3672 & 5.0 \\
\hline Coartem & 3542 & 4.8 \\
\hline Tetanus vaccine & 1862 & 2.5 \\
\hline Tramadol & 1842 & 2.5 \\
Quinine & 1775 & 2.4 \\
Cloxacillin & 1686 & 2.3 \\
Gentamicin & 1602 & 2.2 \\
\hline Ringer's lactate & 1446 & 2.0 \\
\hline
\end{tabular}




\begin{tabular}{cll}
\hline Table 7 Imaging & $\begin{array}{l}\text { Number of } \\
\text { studies }\end{array}$ & $\begin{array}{l}\text { Per cent of } \\
\text { Imaging }\end{array}$ \\
\hline X-ray & 2441 & \\
\hline Chest & 1071 & 43.9 \\
\hline Arm & 477 & 19.5 \\
\hline Leg & 275 & 11.3 \\
\hline Unspecified & 150 & 6.1 \\
\hline Pelvis & 89 & 3.6 \\
\hline Skull & 88 & 3.6 \\
\hline Abdomen & 73 & 3.0 \\
\hline POCUS & 2289 & \\
\hline Unspecified & 1465 & 64.0 \\
\hline FAST & 417 & 18.2 \\
\hline Abdominal & 139 & 6.1 \\
\hline Cardiac echo & 107 & 4.7 \\
\hline Formal US & 1006 & 69.1 \\
\hline Unspecified & 695 & 14.5 \\
\hline Abdominal & 146 & 12.9 \\
\hline Pelvic/obstetric & 130 & \\
\hline
\end{tabular}

FAST, focused assessment with sonography in trauma; POCUS, point-of-care ultrasound; US, ultrasound.

consistent with Uzoechina $e t a l$ 's study from Nigeria and Wachira et al's data from Kenya. ${ }^{56}$

Skills such as X-ray interpretation and POCUS appear to be high yield, given X-rays were performed on $7.0 \%$ and POCUS on $8.2 \%$ of patient visits. POCUS has been shown in other studies to supplement limited radiography capacity and enhance safety of invasive procedures in resource limited settings. ${ }^{23-25}$ The ECP training on POCUS has increased over time and is described elsewhere. ${ }^{26}$

The variety of procedures performed indicates that providers in the setting likely require a broad range of procedural skills. Peripheral intravenous access was the norm, but occasional patients required intraosseous access or cannulation of the external jugular vein. In addition to the common procedures listed above, the ECPs are trained to perform paracenteses, thoracenteses, joint aspirations and nerve blocks. While these procedures are required relatively infrequently, they are crucial for symptomatic relief, making the diagnosis and guiding treatment for a significant proportion of patients seen in rural EDs in SSA. The ability to perform these procedures in a timely manner is required to deliver effective, quality emergency care.

Resource allocation and preparation is the other essential component for effective emergency care delivery. Based on the AFEM framework, Karoli Lwanga Hospital should be an 'intermediate facility'. In the framework, signal functions are defined as 'lifesaving saving clinical interventions,' and a consensus-based list of these functions was generated. While our study did not seek to specifically map which signal functions the ECPs carried out, comparing the list of signal functions with medications administered, procedures performed and laboratory and diagnostic imaging testing used in our cohort, it is clear that there is significant overlap between the framework and the real-world practice of emergency care in this setting.

The medications most frequently used were pain medications, antibiotics, antimalarials, intravenous fluids, nutritional supplements, respiratory medications and cardiac medications. These medications are on the WHO Model List of Essential Medications ${ }^{27}$ and are expected to be widely available. Approximately $13 \%$ of patients received vaccinations as part of their emergency care, highlighting another health systems strengthening role of emergency care, in providing an additional venue for primary prevention. It is also encouraging to see some evidence that patients' pain was addressed, as pain assessment and management has been identified as a deficit in prior studies. ${ }^{6}$

There currently is no widely recognised list of essential emergency laboratory tests in low-resource settings, although the WHO is currently in the process of developing a consensus-based minimum package for this. From our data, it appears that point-of-care blood glucose testing, malaria smears (or rapid diagnostic malaria tests), assessment of haemoglobin, blood typing, HIV testing and fluid analysis (cerebrospinal fluid, urine, pleural fluid, ascites and joint aspirates) are all commonly used. While used to a lesser extent, our database and clinical experience indicated that pregnancy testing is important in this setting as well. The ability to perform HIV screening is critical. Uganda adopted a national guideline recommending opt-out testing in $2005,{ }^{28}$ but missed opportunities for early diagnosis and initiation of antiretroviral treatment in difficult-to-reach populations persist. ${ }^{29-31}$ Due to stock-outs and lack of point-of-care testing performed by ECPs, this cohort of patients had a much lower rate of HIV testing than guidelines recommend. Testing for HIV in the acute care setting offers patients a pathway to access the HIV continuum of care and begin treatment at an earlier stage in the diagnosis, which in turn has been shown to make management more effective and to reduce HIV transmission and the future burden of disease. ${ }^{32} 33$

Future directions for research include comparing data from this facility with training needs and resource allocation at other facilities in SSA. Additionally, formally assessing the use of signal functions in this cohort could help refine the framework proposed to guide emergency care implementation in SSA.

There are several important limitations to this study. It is a single-centre study in rural Uganda with a unique training programme that was developed after a needs assessment of the burden of disease, ${ }^{7}$ but which took into account resource limitations at Karoli Lwanga Hospital. Thus, it is possible that our analysis failed to document 
potentially useful resources that were not available, thus were not part of the ECPs training. Other analyses of smaller portions of this data set looking at common diagnoses can help temper this limitation to some degree. ${ }^{34}$ Practice patterns and proficiencies of the ECPs may not be representative of other clinicians. Increased availability of the X-ray technician would likely have increased the number of patients undergoing imaging in the ED. CT imaging could aid in managing head trauma and some abdominal injuries but is not available at the site; however, this is true in most district hospitals in SSA. Likewise, there may be additional laboratory tests that would be useful but were not available routinely during the study period or were subject to 'stock-outs' of necessary reagents. While we did record tests ordered but not completed, if ECPs were aware of the stock-out, they may not have ordered a test even if they felt was indicated. Given the retrospective nature of this review, we do not have a way of controlling for this lack of ordering due to knowledge of stock-outs.

Free text entry of data by non-medically trained research assistants resulted in a small amount of data being lost due to misspelling or incorrect transcription. Modifications to the data entry process have since reduced such errors. Additionally, patients arriving in the overnight hours when the ED was closed were not included in this analysis. Finally, there was no gold standard arbitration done to assess the true 'need' for the interventions performed or to critique other interventions that might have been indicated. Nonetheless, this provides a pragmatic assessment of the real-world practice of emergency care at a district hospital in a rural area of SSA.

\section{CONCLUSIONS}

This study tracks the resources used to diagnose, stabilise and treat a large cohort of consecutive patients seen in a not-for-profit, rural district ED in SSA. Analysis of the resources used and knowledge and skills applied to care for this patient cohort provides a rare glimpse of what emergency care delivery entails in rural SSA. The results of this study coupled with the previously developed framework to guide emergency care implementation will enable benchmarking and data-driven policy-making for improvement of facility-based emergency care delivery in low-resources settings within SSA.

\section{Author affiliations}

${ }^{1}$ Division of Emergency Medicine, Department of Surgery, Saint Louis University, St. Louis, Missouri, USA

${ }^{2}$ Global Emergency Care

${ }^{3}$ Department of Emergency Medicine, New York University Langone Medical Center, New York City, New York, USA

${ }^{4}$ Harvard Humanitarian Initiative, Harvard University, Boston, Massachusetts, USA

${ }^{5}$ Department of Emergency Medicine, College of Medicine, University of Arizona,

Tucson, Arizona, USA

${ }^{6}$ Department of Health Promotion Sciences, Mel \& Enid Zuckerman College of Public Health, University of Arizona, Tucson, Arizona, USA

${ }^{7}$ Department of Emergency Medicine, CEPA-Idaho, Boise, Idaho, USA

${ }^{8}$ Department of Emergency Medicine, Maine Medical Center, Portland, Maine, USA
${ }^{9}$ Division of Emergency Medicine, Department of Surgery, University of Vermont, Burlington, Vermont, USA

${ }^{10}$ College of Medicine, Mbarara University of Science and Technology, Mbarara, Uganda

${ }^{11}$ Department of Emergency Medicine and the Center for Global Health, University of Illinois, Chicago, Illinois, USA

Acknowledgements Emergency Care Practitioners Felista Kyomugisha, Deus Mubangizi, John Bosco Kamugisha, Elizabeth Natakunda, Pellone Koshaba, Joseph Behangana, Jovita Andinda, Teddie Kire, Irene Nayebare, Alfunsi Birungi, Richard Twinomujuni, Serena Nakato, Hilary Kiiza, Christine Kemirembe, Owen Mugisha, Deus Twinomugisha, and Glorius Kansiime cared for study patients

Contributors MB, SC, UP, HH, BD and SWN designed the database and conceived the study. CCB, BR and UP performed data cleaning, statistical analysis and submission of this work. CCB, SC and UP drafted the manuscript and all authors revised it. SM provided programme support for ECP curriculum development and contributed to study design. All authors take responsibility for the paper as a whole.

Funding This research received no specific grant from any funding agency in the public, commercial or not-for-profit sectors.

Competing interests None declared.

Patient consent Not required.

Ethics approval This study was approved by the Mbarara University of Science and Technology Institutional Review Board, the Uganda National Council for Science and Technology and University of Massachusetts Institutional Review Board (reference number HS 1405). Local approval was obtained from the medical superintendent of the hospital.

Provenance and peer review Not commissioned; externally peer reviewed. Data sharing statement Files will not be available for sharing.

Open Access This is an Open Access article distributed in accordance with the Creative Commons Attribution Non Commercial (CC BY-NC 4.0) license, which permits others to distribute, remix, adapt, build upon this work non-commercially, and license their derivative works on different terms, provided the original work is properly cited and the use is non-commercial. See: http://creativecommons.org/ licenses/by-nc/4.0/

(C) Article author(s) (or their employer(s) unless otherwise stated in the text of the article) 2018. All rights reserved. No commercial use is permitted unless otherwise expressly granted.

\section{REFERENCES}

1 Hirshon JM, Risko N, Calvello EJ, et al. Health systems and services: the role of acute care. Bull World Health Organ 2013;91:386-8.

2 Obermeyer Z, Abujaber S, Makar M, et al. Emergency care in 59 lowand middle-income countries: a systematic review. Bull World Health Organ 2015;93:577-86.

3 Calvello E, Reynolds T, Hirshon JM, et al. Emergency care in subSaharan Africa: results of a consensus conference. Afr J Emerg Med 2013;3:42-8.

4 Wen LS, Char DM. Existing infrastructure for the delivery of emergency care in post-conflict Rwanda: An initial descriptive study. Afr J Emerg Med 2011;1:57-61.

5 Uzoechina NS, Abiola AO, Akodu BA, et al. Pattern and outcome of cases seen at the Adult Accident and Emergency Department of the Lagos University Teaching Hospital, Idi-Araba, Lagos. Nig Q J Hosp Med 2012;22:209-15.

6 Wachira BW, Wallis LA, Geduld H. An analysis of the clinical practice of emergency medicine in public emergency departments in Kenya. Emerg Med J 2012;29:473-6.

7 Periyanayagam U, Dreifuss B, Hammerstedt $\mathrm{H}$, et al. Acute care needs in a rural Sub-Saharan African Emergency Centre: A retrospective analysis. Afr J Emerg Med 2012;2:151-8.

8 World Bank. Rural population. 2016. www.data.worldbank.org/ indicator/SP.RUR.TOTL.ZS

9 Reynolds TA, Calvello EJB, Broccoli MC, et al. AFEM consensus conference 2013 summary: emergency care in Africa - Where are we now? Afr J Emerg Med 2014;4:158-63.

10 Wen LS, Geduld HI, Nagurney JT, et al. Africa's first emergency medicine training program at the University of Cape Town/ Stellenbosch University: history, progress, and lessons learned. Acad Emerg Med 2011;18:868-71. 
11 Kabeza AB, George N, Nyundo M, et al. Development of emergency medicine in Rwanda. Afr J Emerg Med 2013;3:103-9.

12 Busse H, Azazh A, Teklu S, et al. Creating change through collaboration: a twinning partnership to strengthen emergency medicine at Addis Ababa University/Tikur Anbessa Specialized Hospital--a model for international medical education partnerships. Acad Emerg Med 2013;20:1310-8.

13 Martel J, Oteng R, Mould-Millman NK, et al. The development of sustainable emergency care in ghana: physician, nursing and prehospital care training initiatives. J Emerg Med 2014;47:462-8.

14 Hammerstedt H, Maling S, Kasyaba R, et al. Addressing World Health Assembly Resolution 60.22: a pilot project to create access to acute care services in Uganda. Ann Emerg Med 2014;64:461-8.

15 A-Rahman NHA, Jacquet GA. The state of emergency care in the Republic of the Sudan. Afr J Emerg Med 2014;4:55-60.

16 African Federation for Emergency Medicine. Emergency care curriculum. 2016. www.afem.info/resources/afem-curriculum/?id+ $73 \% 27 \mathrm{~A}$.

17 Calvello EJ, Broccoli M, Risko N, et al. Emergency care and health systems: consensus-based recommendations and future research priorities. Acad Emerg Med 2013;20:1278-88.

18 Calvello EJ, Tenner AG, Broccoli MC, et al. Operationalising emergency care delivery in sub-Saharan Africa: consensusbased recommendations for healthcare facilities. Emerg Med $J$ 2016;33:573-80.

19 Uganda Bureau of Statistics 2016. The National population and housing census. 2014. Subcounty Report - Central Region. www. ubos.org

20 United Nations Children's Fund. Uganda country statistics. www. unicef.org/infobycountry/uganda_statistics.html.

21 Rice B, Periyanayagam U, Chamberlain S, et al. Mortality in children under five receiving nonphysician clinician emergency care in Uganda. Pediatrics 2016;137:e20153201.

22 GBD 2015 Mortality and Causes of Death Collaborators. Global, regional, and national life expectancy, all-cause mortality, and causespecific mortality for 249 causes of death, 1980-2015: a systematic analysis for the Global Burden of Disease Study 2015. Lancet 2016;388:1459-544.
23 Sippel S, Muruganandan K, Levine A, et al. Review article: use of ultrasound in the developing world. Int J Emerg Med 2011;4:72.

24 Chavez MA, Naithani N, Gilman $\mathrm{RH}$, et al. Agreement between the World Health Organization algorithm and lung consolidation identified using point-of-care ultrasound for the diagnosis of childhood pneumonia by general practitioners. Lung 2015;193:531-8.

25 Becker DM, Tafoya CA, Becker SL, et al. The use of portable ultrasound devices in low- and middle-income countries: a systematic review of the literature. Trop Med Int Health 2016;21:294-311.

26 Chanler-Berat J, Birungi A, Dreifuss B, et al. Typhoid intestina perforation: Point-of-care ultrasound as a diagnostic tool in a rural Ugandan Hospital. Afr J Emerg Med 2016;6:44-6.

27 World Health Organization. WHO Model List of Essential Medications. 2015

28 Uganda Ministry of Health. Uganda national policy guidelines for HIV counseling and testing. 2005. www.who.int/hiv/pub.guidelines/ uganda_art.pdf (accessed 25 Sep 2016).

29 Nakanjako D, Kamya M, Daniel K, et al. Acceptance of routine testing for HIV among adult patients at the medical emergency unit at a national referral hospital in Kampala, Uganda. AIDS Behav 2007;11:753-8

30 Larsson EC, Thorson A, Pariyo G, et al. Opt-out HIV testing during antenatal care: experiences of pregnant women in rural Uganda. Health Policy Plan 2012;27:69-75.

31 Obermeyer CM, Bott S, Bayer R, et al. HIV testing and care in Burkina Faso, Kenya, Malawi and Uganda: ethics on the ground. BMC Int Health Hum Rights 2013;13:6.

32 Donnell D, Baeten JM, Kiarie J, et al. Heterosexual HIV-1 transmission after initiation of antiretroviral therapy: a prospective cohort analysis. Lancet 2010;375:2092-8.

33 Gardner EM, Haukoos JS. At the crossroads of the HIV Care Continuum: emergency departments and the HIV Epidemic. Ann Emerg Med 2015;66:79-81.

34 Chamberlain S, Stolz U, Dreifuss B, et al. Mortality related to acute illness and injury in rural Uganda: task shifting to improve outcomes. PLoS One 2015;10:e0122559. 